\title{
Measurement of Pure Shear Constitutive Relationship from Torsion Tests under Quasi-static, Medium and High Strain Rate Conditions
}

\author{
Longhui Zhang $^{1 *}$, David Townsend ${ }^{1}$, Nik Petrinic ${ }^{1}$, Antonio Pellegrino ${ }^{1}$ \\ ${ }^{1}$ Department of Engineering Science, University of Oxford, Parks Road, Oxford, \\ OX1 3PJ, U.K. \\ *Corresponding author: longhui.zhang@eng.ox.ac.uk
}

\begin{abstract}
:
Torsion tests provide important shear stress and shear strain relationships to reveal the fundamental plastic flow response of a material. Bespoke torsion techniques complemented by Digital Image Correlation are developed to accurately measure the shear stress-strain relationship at quasi-static, medium rate $9 / \mathrm{s}$ and high strain rate above 1000/s. The equipment used includes a screw driven mechanical system, a hydraulic Instron machine and a Campbell thin-walled tube split Hopkinson torsion bar equipped with an ultra-high speed camera. A near alpha Ti3Al2.5V alloy was used as a model material in this study. A four camera digital image system has been constructed to monitor the material deformation and failure during a low rate torsion test, to gain further insight into plastic deformation of the tubular specimen. Shear stress-strain relationship of the $\mathrm{Ti} 3 \mathrm{Al} 2.5 \mathrm{~V}$ alloy exhibits noticeable strain rate sensitivity. Observations of the strain hardening rate evolution indicate that the hardening capacity of $\mathrm{Ti} 3 \mathrm{Al} 2.5 \mathrm{~V}$ is both strain and strain rate dependent. High strain rate torsional stress-strain relationship shows lower strain hardening, compared to the response obtained from a shear compression specimen. The present techniques are demonstrated to be suitable for the measurement of pure shear constitutive relationship, including rate sensitivity and failure of the material.
\end{abstract}

Keywords: Constitutive behavior, Pure Shear, Stress Wave, Localization, Titanium alloys, Hopkinson bar 


\section{Introduction}

During the life of a jet engine, the fan blade and engine containment are inevitability subjected to impact loading in the events such as the blade off and bird strike, in which the material and structures experience high strain rate deformation process. The constitutive relationship over a range of strain rates is required for the analysis of structural response under these dynamic impact situations.

The flow stress of many materials increases with the increase of high strain rate, which is important for the study of failure mechanisms and dynamic constitutive relationship for engineering materials. The Split Hopkinson Pressure Bar (SHPB), initially designed by Hopkinson [1] and later Kolsky [2], has been effective for the measurement of stress, strain and strain rate relations under uniaxial stress state during rapid deformation process. The basis of SHPB technique is the specimen is sandwiched between the incident bar and transmitted bar. The incident bar was impacted by a striker bar to generate a compressive stress wave propagating along the bar towards the specimen. The last several decades have seen many improvements and developments in SHPB technique [3-6]. Because of the dispersion effect during wave propagation, inertia effect and the friction effect between specimen and two loading bars [7-9], the analysis of SHPB experiments is relatively complicated including some correction factors. Furthermore, the fluctuation amplitude could reach $20 \%$ of the wave amplitude [10], due to the radial expansion of the specimen.

Given the above reasons, the Torsion Split Hopkinson Bar (TSHB) was proposed by Baker and Yew [11] in 1966. Shortly after Campbell et al. [12, 13] at Oxford University, jointly with Duffy et al. [14] at Brown University and Nicholas et al. [15-17] in the Air Force Research Laboratory, made significant improvements in the generation of torsional wave and the TSHB experimental setups. The experimental and theoretical analysis showed that there was no dispersion in the propagation of torsion wave in the round bar when the initial condition was a linear function of cylindrical radial coordinate. Without the existence of radial motion, the radial inertial effect and the friction effect are absent. 
Due to the simplicity of the compression test, most of the high strain rate constitutive relation has been obtained using the spilt Hopkinson compression bar [18-21], which provides constitutive response under assumed uniaxial stress condition. To achieve high strain rates, the specimen gauge length needs to be reduced, which could increase the potential errors resulting from the friction effect. Meanwhile, a constitutive equation is desirable to take into account stress states instead of uniaxial stress conditions. The biaxial stress ratio of -1 in torsion test at quasi-static and high strain rates, which is equal to pure shear $[22,23]$, is of a fundamental significance to reveal the plastic flow response of a material, compared to the measured stress-strain relationship under uniaxial stress state condition. A noticeable exception is the design of shear compression specimen by Rittel et al. [24-27], to allow large strain testing of materials over wide ranges of strain rates under complex stress state conditions.

Titanium alloys with high strength to weight ratio and corrosion resistance have attractive properties for aerospace engineering applications [28]. The ability to design components, which suffer high-speed deformation process due to impact events such as bird strike, is of vital importance. Macdougall and Harding [29, 30] studied the constitutive relationship of Ti6Al4V alloy at low and high strain rates using the standard Campbell (or Duffy) thin-walled tube Hopkinson torsion bar. The adiabatic heating characteristic at high strain rates was also reported by using transient infrared thermometry technique and high-speed photography technique. Similarly, Liao and Duffy [31] carried out a series of dynamic torsional experiments with the main emphasis on the initiation and formation of adiabatic shear bands in Ti6Al4V alloy. Chichili et al. [32] studied the temperature and strain rate sensitivity of commercially pure titanium at high strain rates, and the adiabatic shear localization by using the torsional Hopkinson bar and the modified compression-torsion Hopkinson bar. Yang et al. [33] connected dynamic shear stress-strain relations and microstructural evolution in Ti6Al4V alloy in a modified Hopkinson torsion bar which provided a single loading torsional stress pulse to interrupt the high rate tests and consequently freeze the material microstructure formed during the effective period of loading in the specimen. Most of the studies of torsion tests were carried out in the high strain rate 
regions, and most of the tests of titanium alloys were limited in Ti6Al4V alloy or pure titanium. Recently, Zhang et al. [34, 35] investigated the dominated shear response and the corresponding real time adiabatic temperature rise of several aircraft structural titanium alloys such as Ti3Al2.5V alloy. However, investigations of Ti3Al2.5V alloy under pure shear condition are still limited. Given that the engineering applications of titanium alloys are to resist dynamic loading, it is understandable that the previous pure shear characterization has been mainly at high strain rates, however, the medium strain rate data, which is rarely reported in the literature, is inherently important to provide a better understanding of the rate dependency under pure shear conditions. In the present paper, newly designed torsion experiments from quasi-static condition, medium to high strain rates, complemented by DIC with noncontact characteristics $[33,36,37]$, are carried out to study the shear stress-strain relationship of a near $\alpha$ Ti3A12.5V alloy by using the tubular specimen. This alloy was chosen as a model material because of its excellent strength-to-weight ratio combined with its good cold formability. The combined high-speed DIC technique and experimental apparatus can be used to accurately measure the pure shear response at various strain rates and consequently will benefit the design of impact resistant turbine engine containment component. The present work has the following goals:

- Designing the testing apparatuses for pure shear characterization for the quasi-static condition, medium strain rates and high strain rates

- Providing a better understanding of the global and local shear stress-strain relation from the thin-walled tubular specimen

- Studying the constitutive response and failure of Ti3Al2.5V alloy at various constant strain rate regions

The paper is therefore organized as follows: the material, geometries of the torsion specimen and the experimental setups are introduced in Section 2. The experimental results at various strain rates are reported in Section 3. The next section is the discussion and summary of the experimental data, following by concluding remarks. 


\section{Experiment Protocol}

\subsection{Materials and specimen}

The thin wall tubular specimens shown in Fig. 1 were manufactured from a $35 \mathrm{~mm}$ diameter rod of the Ti3A12.5V titanium alloy. The specimen has a gauge length of 2.5 $\mathrm{mm}$ and a wall thickness of $0.30 \pm 0.01 \mathrm{~mm}$ with an internal diameter of $13.8 \mathrm{~mm}$. For subsequent DIC analysis of the shear strain distribution during deformation, a speckle pattern was applied to the gauge section of the specimen with a fine spray of black ink over a solid white ink coating. The flanges of the specimen are designed to be a good fit into matching hexagonal sockets in the mechanical testing apparatus.

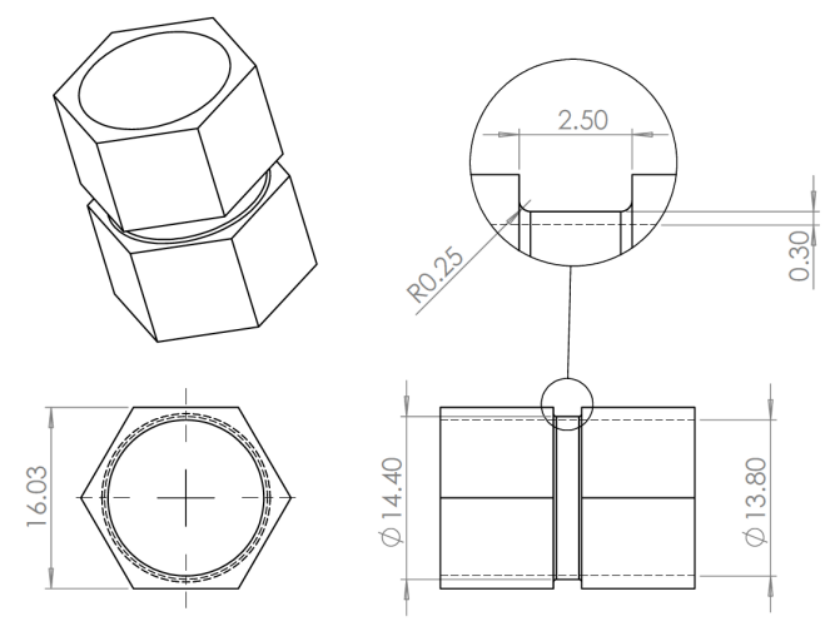

(a)

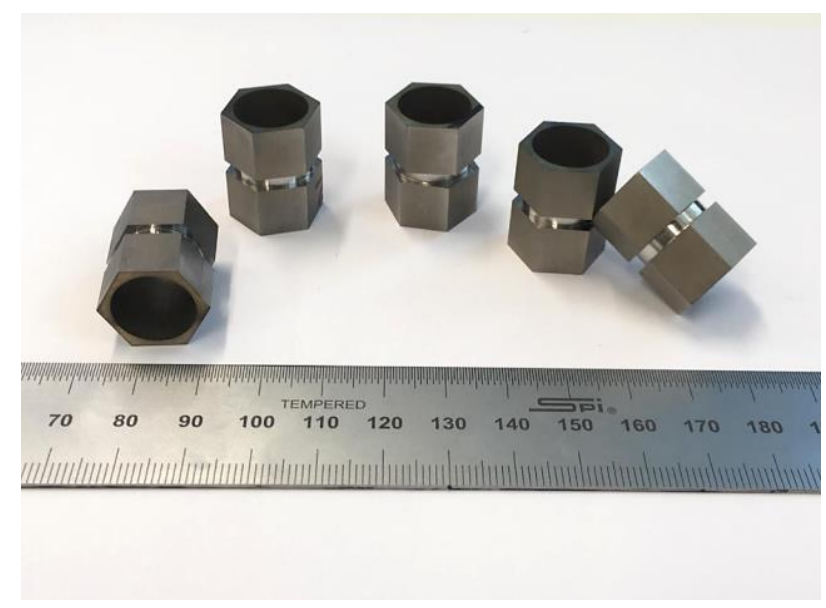

(b)

Fig. 1 The tubular specimens (a) Geometry and dimensions (b) Image of the specimens 
Torque data recorded by the Zwick machine, hydraulic Instron machine and Hopkinson bar during deformation were converted into shear stress according to

$$
\tau=\frac{\mathrm{rT}}{\mathrm{J}}
$$

where $\tau$ is the shear stress, $\mathrm{r}$ is the average of inner and outer gauge section radii, $\mathrm{T}$ is the torque. $\mathrm{J}$ is the polar moment of inertia. Angle of twist data recorded during deformation was converted into global engineering shear strain using

$$
\gamma_{\text {global }}=\frac{\mathrm{r} \theta}{l}
$$

where $\gamma_{\text {global }}$ is the global engineering shear strain from the machine measurement, defined as such so it is distinct from the local engineering shear strain determined with DIC, $\theta$ is the angle of twist and $l$ is the shear gauge length, equal to $2 \mathrm{~mm}$ here.

\subsection{Experimental setup}

\subsubsection{Quasi-static testing}

In previous studies $[22,38]$, quasi-static tests had been performed by using an electric motor connected to the Hopkinson bar. In this work, the quasi-static torsion technique was developed and the torsion tests were conducted on a Zwick-Roell Z250 machine, synchronized with four IDS (Image Development Systems) UEye USB 3.0 Cameras ${ }^{1}$ with high resolution images (2456x2054 pixels) at a frame rate of 1 fps to observe the speckle painted specimen gauge section during the test. The speed of the mechanical driven actuator was programmed to produce a constant angular velocity of $0.002 \mathrm{rad} / \mathrm{s}$ corresponding to an observed shear strain rate in the specimen of approximately 0.005 /s. The stress in the test specimens is calculated from torque measurements recorded by the test machines during the tests and the strain obtained from direct measurement of the specimen surface displacement using digital image correlation techniques. These images were analysed using digital image correlation software (Lavision

\footnotetext{
${ }^{1}$ IDS Imaging Development Systems GmbH, Dimbacher Str. 6-8, 74182 Obersulm, Germany
} 
$\mathrm{DaVis}^{2}$ ) in order to obtain the shear strain in the specimen during the test. Note that there exists a measurement uncertainty using two dimensional DIC for the analysis of shear strain on a cylindrical surface, as shown in Appendix A. Given that the error is less than $1 \%$ in the range of shear strain values in the present work, no correction is used in the shear strain from DIC analysis. The four cameras were placed with the angular separation between each camera approximately $90^{\circ}$, as shown schematically in Fig. 2. The cameras were synchronised and the images recorded using the commercially available StreanPix digital video recording software.

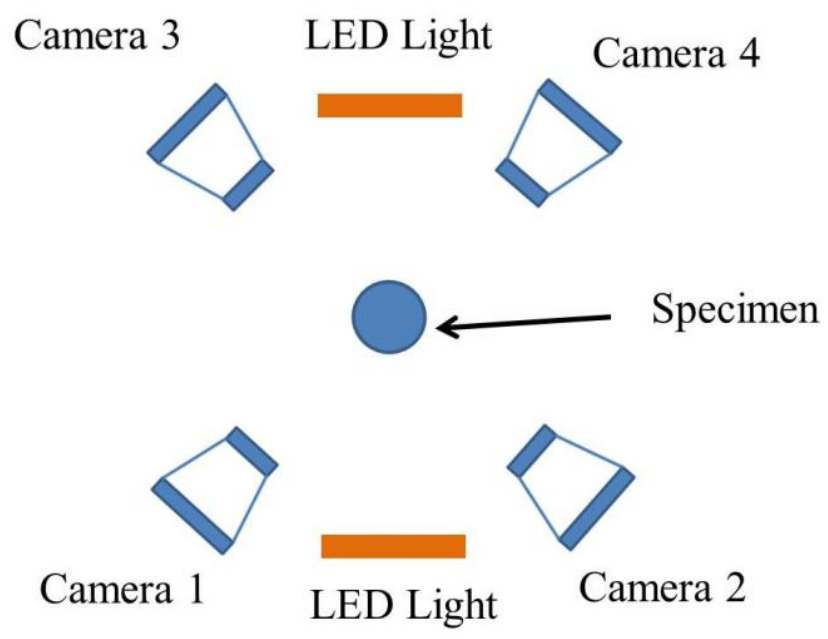

Fig. 2 Camera configuration diagram in low rate torsion experimental setup

\subsubsection{Medium strain rate testing}

The configuration for the medium strain rate torsion tests was based on the one employed for the low rate torsion tests, except that a high speed rotary actuator was used. The torsion specimen fixtures were attached to the rotary actuator and the dynamic torque load cell of the Instron 8854 test machine, as shown schematically in Fig. 3. The accurate strain measurements in the specimen were obtained using DIC techniques on a series of high speed images with resolution of 512x296 pixels recorded with a Photron camera at a framing rate of 10,000 fps. The torque signals

${ }^{2}$ LaVisionUK Ltd, 2 Minton Place, Victoria Road, Bicester OX26 6QB, United Kingdom 
from the Instron machine were recorded with an acquisition rate of 10,000 Hz. The torsion fixture attached to the machine has a hexagonal cut out in the end. The hexagonal torsion specimen is inserted into the cut out. The upper part of the fixture and specimen rotate to the right during the test whilst the lower part is fixed, producing shear in the gauge section. The speed of the actuator was programmed to produce a nominal shear strain rate of $9 / \mathrm{s}$ in the gauge section of the test specimen. Great care was taken during the setting up of the equipment and during the test programme to ensure accurate axial alignment of the fixtures and test specimen.

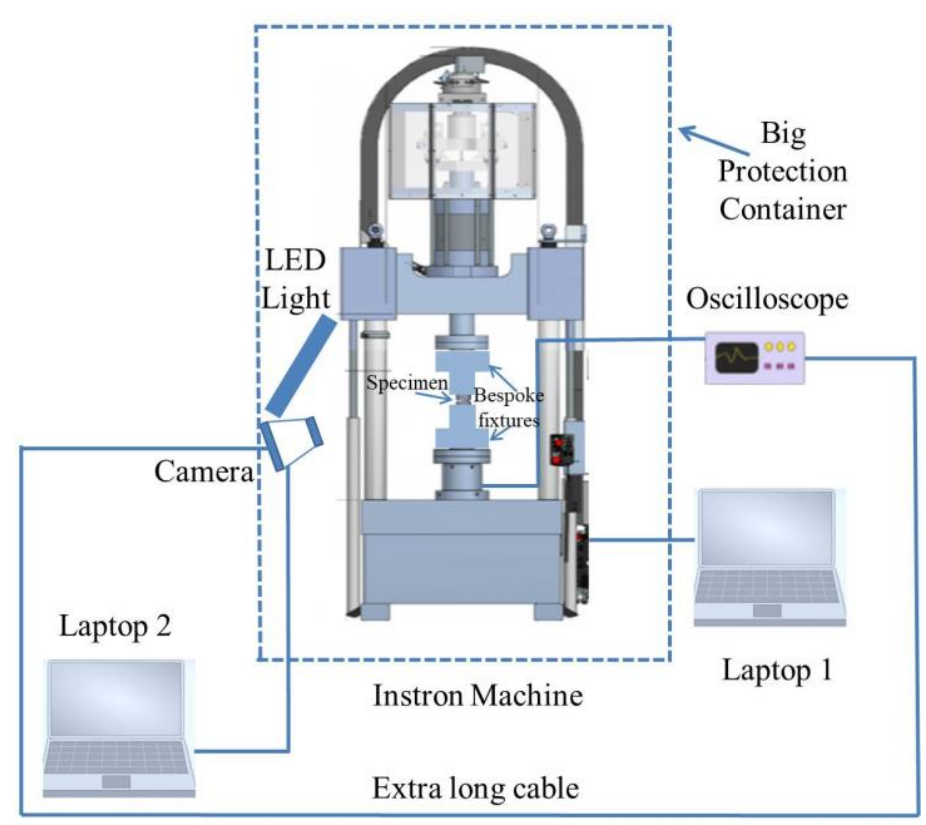

Fig. 3 Diagram of test setup at medium strain rate of 9/s based on the Instron machine

\subsubsection{High strain rate testing}

For high rate torsion tests, the Split Hopkinson torsion bar was employed which was initially designed by Campbell et al. [12, 13] and had been used previously for dynamic characterization of metals, polymers and foams [22, 23, 29, 38]. Stored torque in the incident bar is applied to the tubular specimen on the rapid release of a fixing clamp. The hexagonal ends of the thin-walled specimen are placed in matching hexagonal cuts of the incident and transmitted bars. The diagram of the Hopkinson 
torsion bar setup, together with the stress wave propagation is shown in Fig. 4. The motor at the right end, which was previously used for quasi-static torsion tests, is not connected to the transmitted bar. The deformation of the specimen was observed with an ultrafast Kirana camera. The high-speed images recorded by using a Tamron SP60mm Macro lens mounted on the Kirana camera have a resolution of $924 \times 748$ pixels, at framing rates from $2 \times 10^{5} \mathrm{fps}$ and shutter speed 5 us.

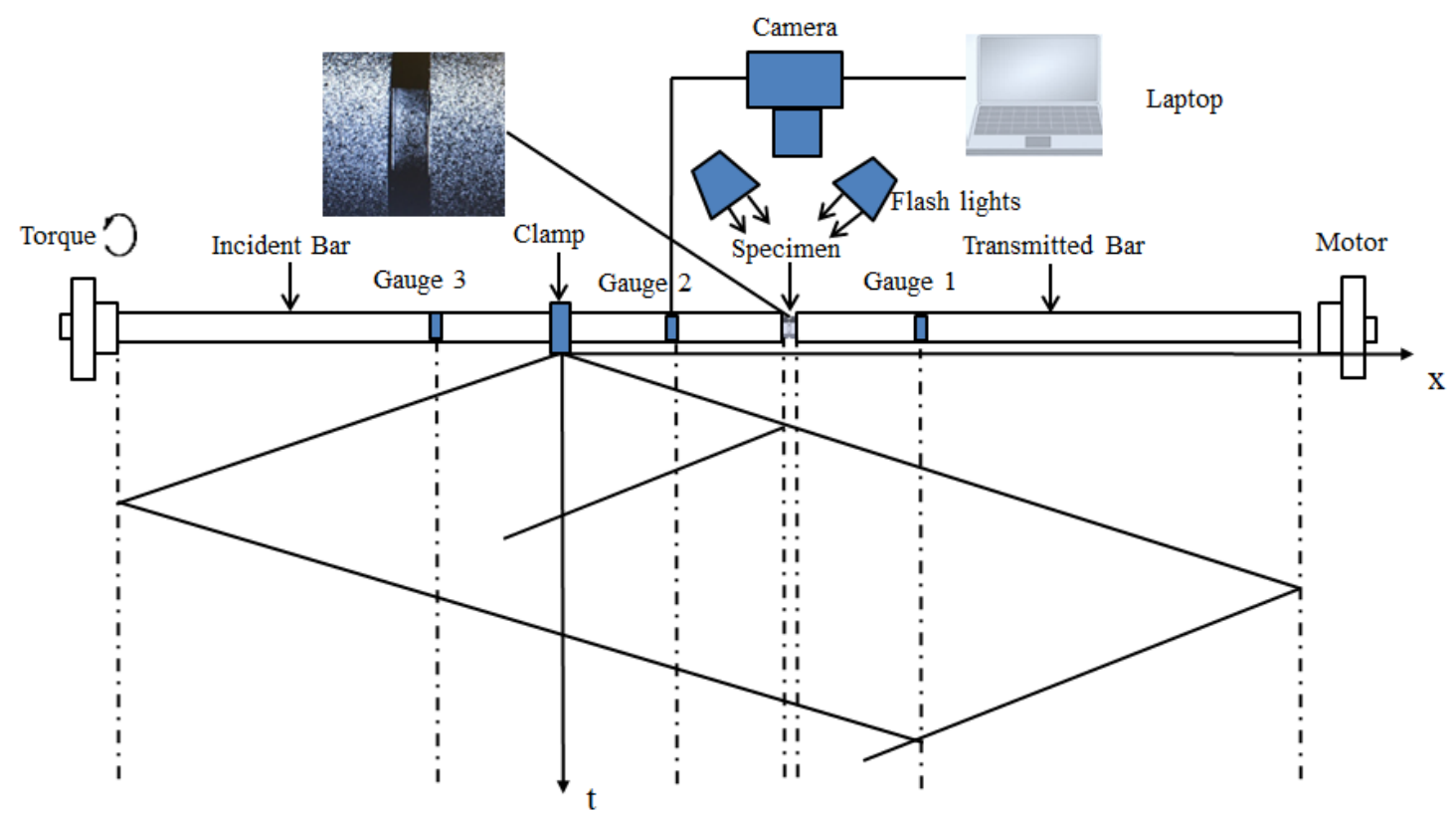

Fig. 4 Diagram of Hopkinson torsion bar along with the stress wave propagation.

The Hopkinson torsion bar comprises incident and transmitted bars of $2.4 \mathrm{~m}$ in length and $25.4 \mathrm{~mm}$ in diameter made from Ti6Al4V alloy. A stored torque in the incident bar propagates along the bar and applies to the specimen after the rapid release of a fixing clamp. This Hopkinson bar setup was used to achieve shear strain rates at the level of approximately 1000 /s. Strain gauge 1 and 2 are attached on the transmitted bar and incident bar respectively. To obtain the deformation information across the gauge section, the real time recorded high-speed images are analyzed by DIC for the direct shear strain measurement. A typical incident and transmitted pulse from the Hopkinson torsion bar apparatus are shown in Fig. 5a. The torque equilibrium at both ends of a specimen is important for a valid torsional experiment on the bar. Fig. $5 b$ 
shows the toque at both ends of a specimen as a function of time. Although there is a first period when the potential slack [22] is taken up, dynamic equilibrium at both ends has been established once the plastic region is entered. The output torque is used for the shear stress measurement of the specimen.

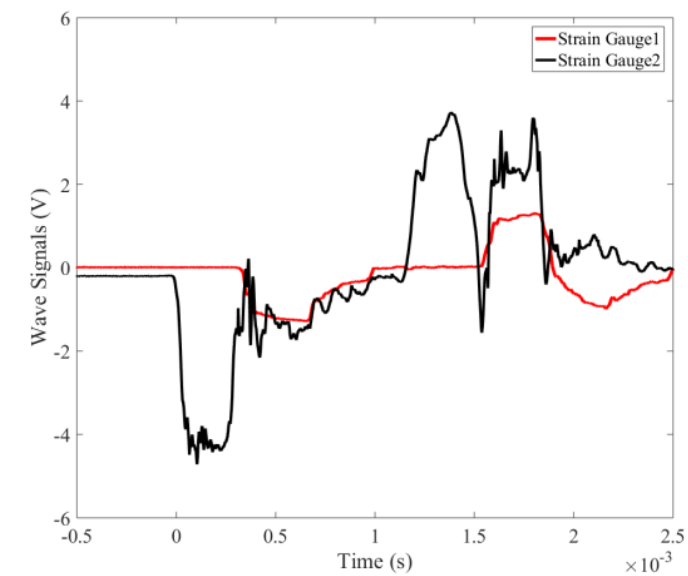

(a) Strain gauge signals

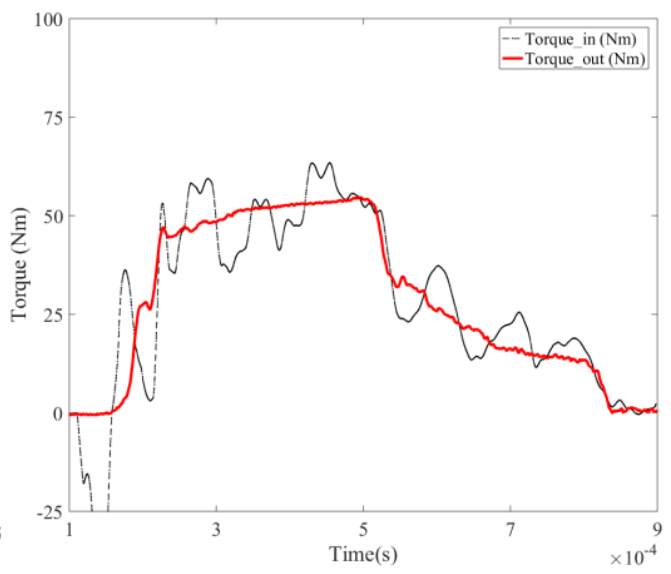

(b) Dynamic torque equilibrium

Fig. 5 Typical measurements at high strain rate of 1600/s on the Hopkinson bar

\section{Experimental Results}

\subsection{Quasi-static torsion test results}

The torsion test configuration generates shear strain around the circumference of the specimen. The deformation process was monitored by a four camera system to achieve shear strain measurements around the circumference of the specimen simultaneously. The typical recording from camera 1 from the test 1 is presented in Fig. 6. Fig. 6a shows the image of the specimen at the start of the test, Fig. 6b-d demonstrate that this test configuration enables identification of the failure location highlighted by the arrows in the image from Fig. 6d. The shear deformation in the specimen is evident from the distortion of the speckle pattern. The shear strain from each camera was calculated in the specimen at the centre of the image over a $2 \mathrm{~mm}$ gauge length in DIC analysis. 

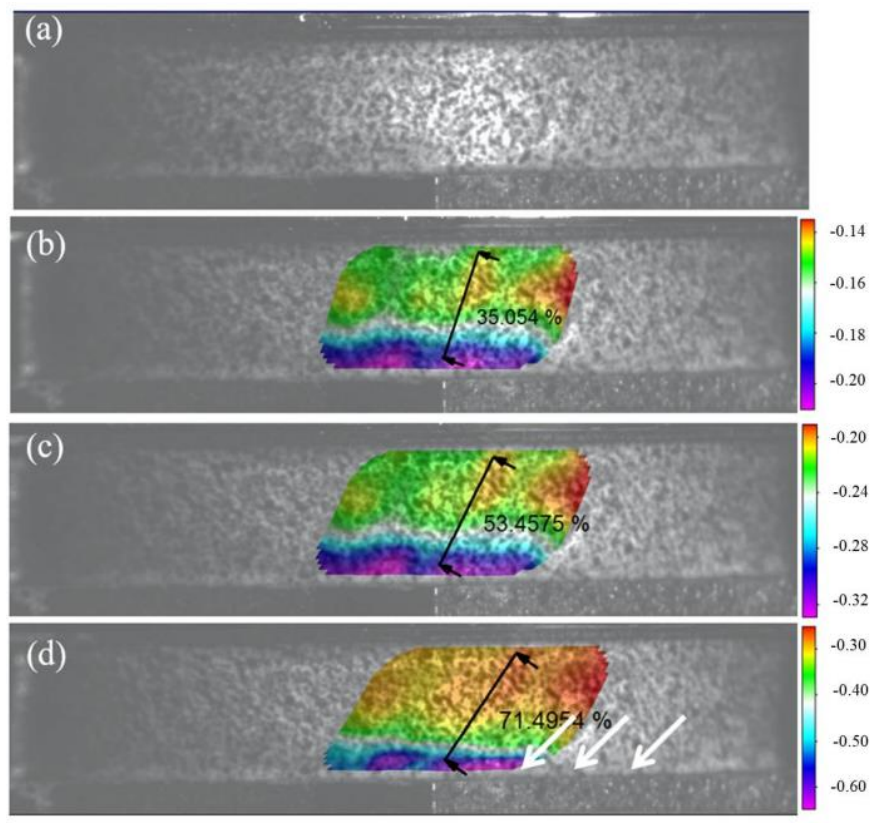

Fig. 6 Camera 1 images with shear strain contours from DIC at different deformation stages (a-d). Failure in image (d) is arrowed.

It is important to note, as shown in Fig. 7, that although failure of the material can be seen in the image from camera 1 or 2 , it is not evident in the images from the other cameras taken at the same time.
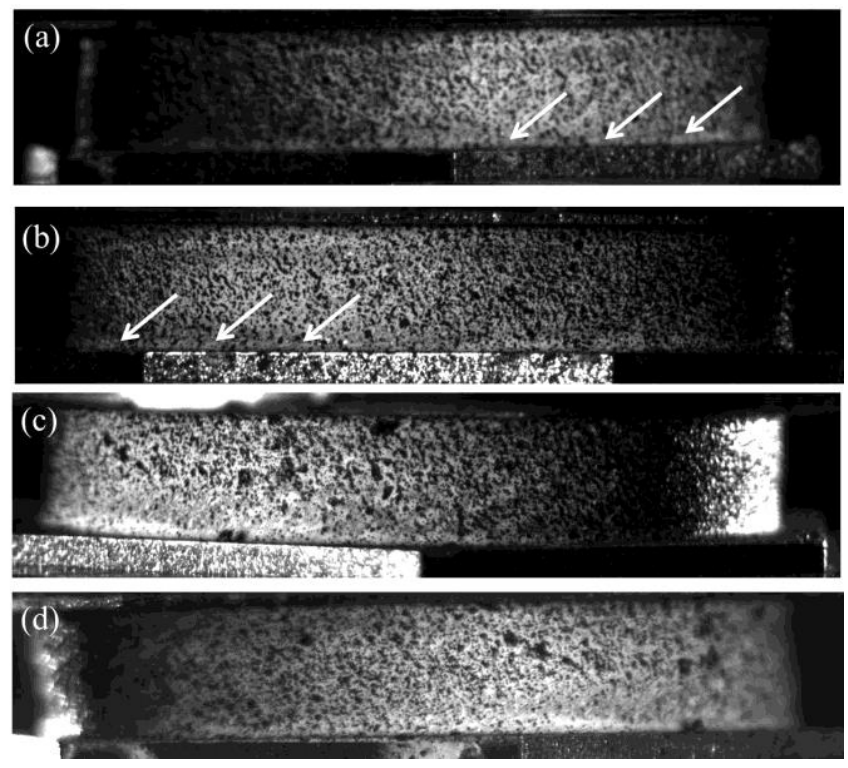

Fig. 7 Images from each of the four cameras at onset of failure for torsion specimen.

(a) Image from camera 1, (b) Image from camera 2, (c) Image from camera 3, (d) Image from camera 4. 
Fig. 8 a shows the typical shear stress and the corresponding shear strain histories from each camera recording. The initial shear strains measured at each camera location are consistent indicating an initial elastic response followed by increasing plastic strain. With the increase of strain, some variations in the magnitude of the shear strain can be seen. At the point of failure, indicated by the load drop in the shear stress at approximately $127 \mathrm{~s}$, the shear strain varies from 0.59 to 0.70 . These results indicate that there are some differences in the strain distribution around the circumference of the specimen.

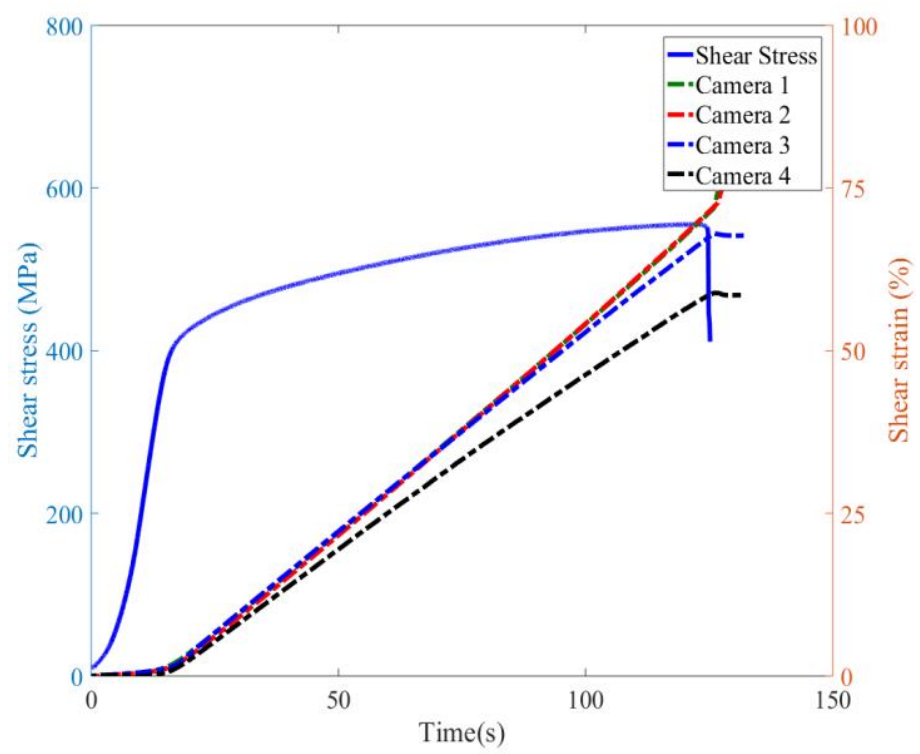

(a)

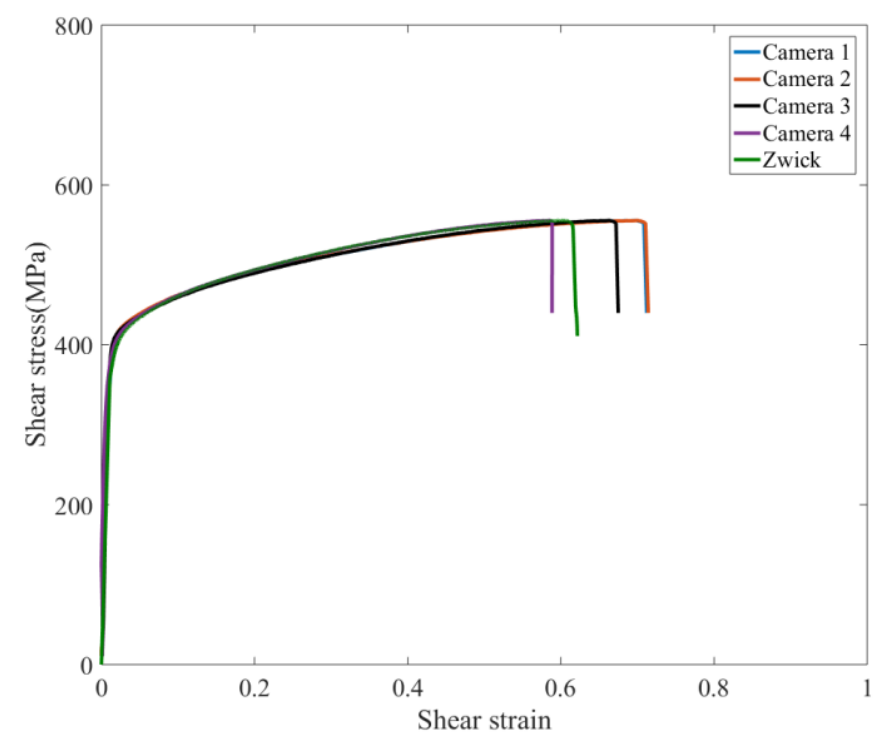

(b) 
Fig. 8 Typical low rate torsion test results (a) Shear strain and stress as a function of time (b) Shear stress-strain relationships measured from four cameras and the Zwick machine

The shear stress of the specimen is calculated from the global torque applied to the specimen as recorded by the test machine. Clearly this represents the average shear stress in the test section of the specimen. The shear stress-strain relationships measured from four cameras and the Zwick machine were obtained as shown in Fig. $8 \mathrm{~b}$. The test machine data has been analysed to provide a global measurement of the engineering shear stress and shear strain experienced by the specimen. Clearly the Zwick based stress-strain data is in good agreement with data obtained by DIC technique for the response of the material. The Zwick data, however, does not represent the localised failure strain as it represents the overall behaviour of the specimen. For each specimen, good agreement is seen at the four different measurement locations for the initial elastic and plastic response of the material up to the onset of failure.

The specimens from three low rate torsion tests are evaluated by comparing the flow stress at a shear strain value of 0.3 as shown in Table 1 . It is found that flow stress is in good agreement for each specimen. Considering the failure, different failure strains are observed in the material at each camera location as summarised in Table 2. For instance, the failure strain (strain at load drop) values from camera 1 and 2 are 0.70 in test 1 , which is higher than the failure strain from camera 3 and 4 with the values 0.66 and 0.59. The global failure strain from the Zwick machine is also added into for a comparison. It is observed that the average failure strain 0.66 from 4 camera system agrees with the global failure strain 0.60 , the former being $9 \%$ higher than the macro measurement. 


\begin{tabular}{|c|c|c|c|}
\hline \multirow{2}{*}{ Camera } & \multicolumn{3}{|c|}{ Flow stress (MPa) } \\
\cline { 2 - 4 } & Test 1 & Test 2 & Test 3 \\
\hline Camera 1 & 512 & 516 & 515 \\
\hline Camera 2 & 512 & 515 & 514 \\
\hline Camera 3 & 512 & 509 & 512 \\
\hline Camera 4 & 517 & 512 & 511 \\
\hline Average & $513 \pm 2.5$ & $513 \pm 3.2$ & $513 \pm 1.8$ \\
\hline Zwick & 517 & 517 & 516 \\
\hline
\end{tabular}

Table 1 Quantitative comparison of flow shear stress at 0.3 shear strain from 4 camera system and the Zwick Machine. The average shear stress value from 4 camera system is given with the standard deviation.

\begin{tabular}{|c|c|c|c|}
\hline \multirow{2}{*}{ Camera } & \multicolumn{3}{|c|}{ Failure engineering strain } \\
\cline { 2 - 4 } & Test 1 & Test 2 & Test 3 \\
\hline Camera 1 & 0.70 & 0.50 & 0.55 \\
\hline Camera 2 & 0.70 & 0.50 & 0.58 \\
\hline Camera 3 & 0.66 & 0.57 & 0.57 \\
\hline Camera 4 & 0.59 & 0.54 & 0.61 \\
\hline Average & $0.66 \pm 0.05$ & $0.53 \pm 0.03$ & $0.58 \pm 0.03$ \\
\hline Zwick & 0.60 & 0.49 & 0.54 \\
\hline
\end{tabular}

Table 2 Quantitative comparison of failure strain from 4 camera system and the Zwick Machine. The average failure strain value from 4 camera system is given with the standard deviation. 
As the shear stress increases during the test, some degree of non-uniformity is observed in the shear strain distribution. Note that the initial failure is a localised event. The initial failure or crack is only observed at one (or two) location and can be identified with the assistance of high resolution images. Therefore, the shear stress-strain behaviour of the material can be obtained from the failure location. Table 3 shows the failure location identified from the 4 camera system. The local shear stress-strain responses for each test are shown in Fig. 9. Note that this local shear stress-strain relationship is regarded as engineering shear stress-strain relationship of the material, which is the average response across the $2 \mathrm{~mm}$ gauge length in the area of gauge section where failure locates. The local shear stress strain relationship presents similar behaviour with a yield stress of approximately $375 \mathrm{MPa}$. The material failure strain lies in the range of 0.57 to 0.70 .

\begin{tabular}{|c|c|c|}
\hline Specimen Test & Failure Location & Shear Strain at Failure \\
\hline Test 1 & Camera 1 or 2 & 0.70 \\
\hline Test 2 & Camera 3 & 0.57 \\
\hline Test 3 & Camera 4 & 0.61 \\
\hline
\end{tabular}

Table 3 Shear strain at onset of failure at identified failure location.

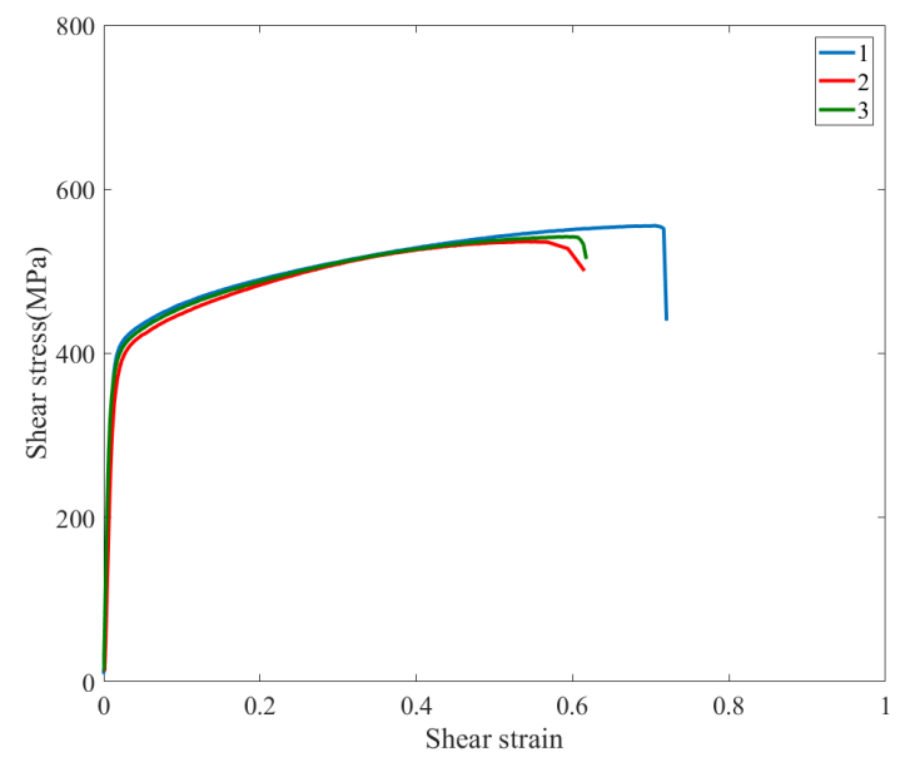


Fig. 9 Local shear stress vs shear strain for low rate torsion tests from identified failure locations. Note that this local shear stress-strain relationship is the average response across the $2 \mathrm{~mm}$ gauge length in the area of gauge section where failure locates.

\subsection{Medium strain rate torsion test results}

The medium strain rate tests were carried out at a strain rate of approximately $9 / \mathrm{s}$. Fig. 10 shows the shear stress and shear strain rate evolution measured from the test machine and DIC analysis, together with the deformation process at different stages. The torque measurement from the sensor fixed on the base of the Instron machine shows oscillations because of the elastic response and the inertia of the measurement system [39, 40]. A constant strain rate is achieved when the specimen enters the plastic deformation region. The shear stress suddenly drops at stage 3 , followed by the final fracture (white arrow) of the specimen.

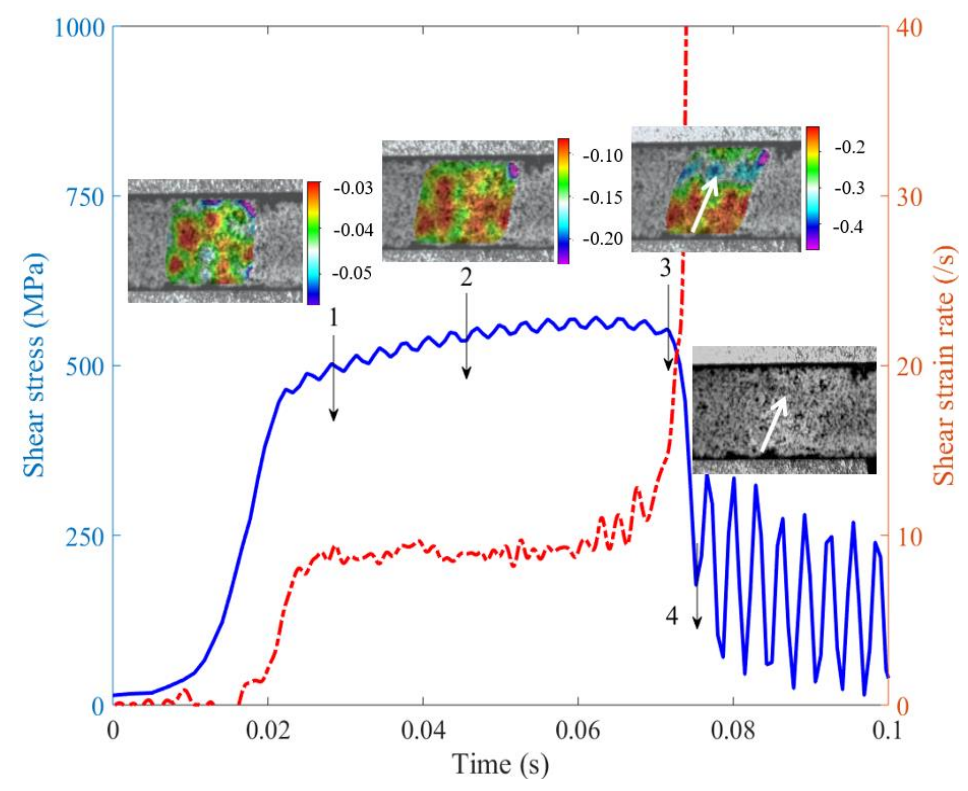

Fig. 10 Typical shear stress and shear strain rate histories and the corresponding deformation process (engineering shear strain is shown) at strain rate of $9 / \mathrm{s}$

\subsection{High strain rate torsion test results}

Fig. 11 shows the shear stress history recorded on the Hopkinson torsion bar system, together with the corresponding shear strain rate as a function of time using a numerical low-pass filter [41] in MATLAB. Typical deformation process at various 
stages is also given. An almost constant strain rate of $1600 / \mathrm{s}$ is achieved in the plastic deformation region. At the load drop point around 336 us, the strain rate soars up indicating the rapid failure process in the specimen. The incident bar is at the left side, and the rotation of the specimen connected to the incident bar and the shear deformation of the gauge section can be seen. The shear stress drops rapidly beyond stage 3, while the simultaneous photograph indicates the formation of localized shear band marked by the while arrow associated with the loss of the load bearing capacity. This is followed, very quickly, by final fracture of the specimen.

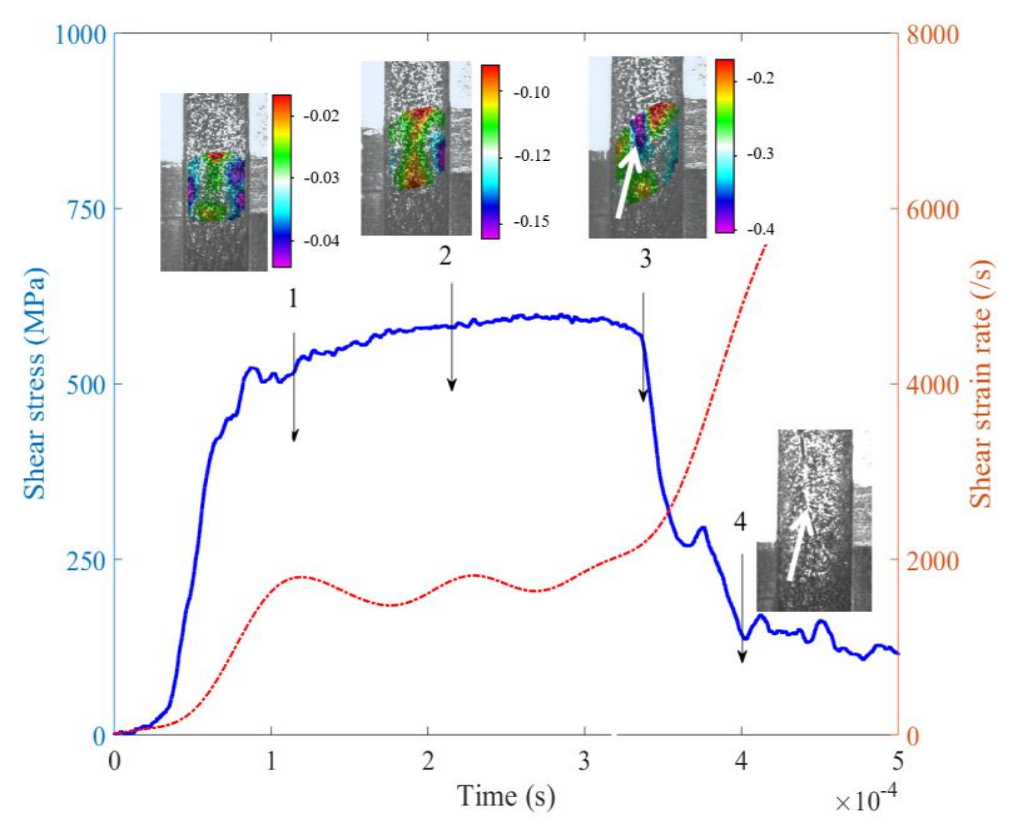

Fig. 11 Typical shear stress and shear strain rate histories and the corresponding deformation process (engineering shear strain is shown) at strain rate of 1600/s

The shear stress-strain relationship obtained from the fracture region observed with the high-speed camera on the circumference of the specimen is presented in Fig. 12 for three tests, together with the quasi-static and medium strain rate data. The shear yield strength values at three different strain rate regions are given in Table 4 . The shear constitutive response at high strain rates of 1200 /s-1600/s shows good repeatability with an average shear yield stress of approximately $445 \mathrm{MPa}$ and shear failure strain of approximately 0.45 . 


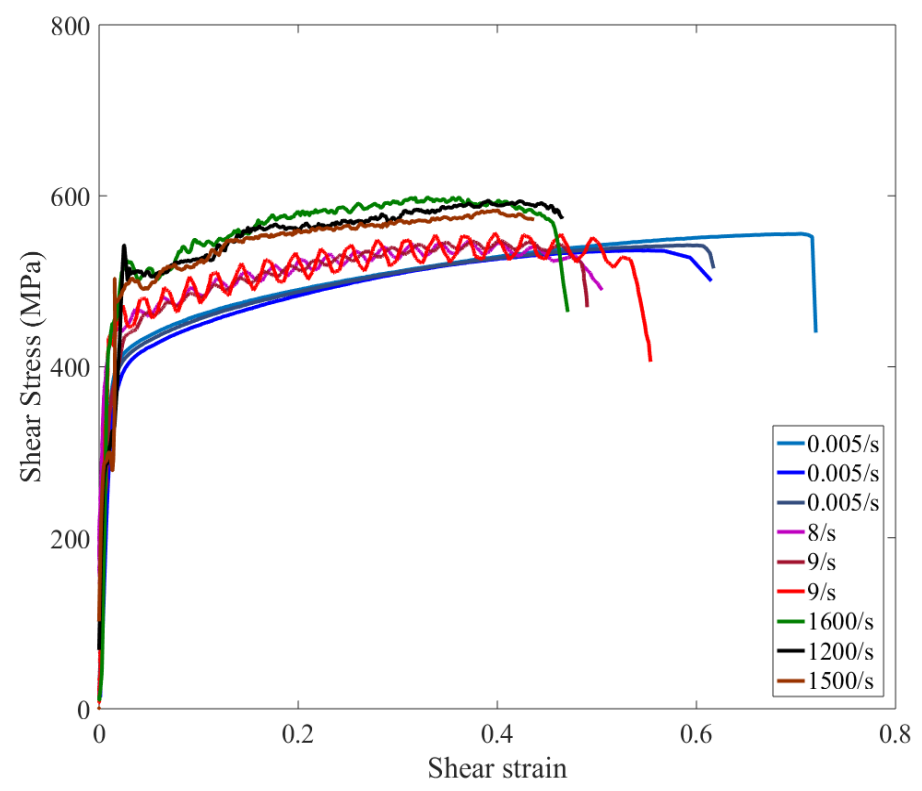

Fig. 12 Comparison of shear stress-strain relationship from low to high strain rates

\begin{tabular}{|c|c|c|c|}
\hline \multirow{2}{*}{ Strain rate (/s) } & \multicolumn{3}{|c|}{ Yield Stress (MPa) } \\
\cline { 2 - 4 } & Test 1 & Test 2 & Test 3 \\
\hline 0.005 & 375 & 375 & 373 \\
\hline $8-9$ & 405 & 390 & 405 \\
\hline $1200-1600$ & 440 & 445 & 450 \\
\hline
\end{tabular}

Table 4 Shear yield strength values from three tests under quasi-static, medium and high strain rate conditions.

The increasing trend of shear stress can be seen across the strain rates between 0.005 /s to 1000 /s. The medium strain rate data, which cannot be easily achieved with conventional loading devices, is important to illustrate the trend of shear stress as a function of logarithmic shear strain rate. Here, the Cowper-Symonds [42] material model is employed to describe the rate dependency of shear stress,

$$
\tau=\tau_{0}\left[1+\left(\frac{\dot{\gamma}}{D}\right)^{1 / p}\right]
$$

where $\tau_{0}$ is the average shear stress at shear strain rate of $0.005 / \mathrm{s}, \dot{\gamma}$ is the strain rate, $\mathrm{D}$ and $\mathrm{p}$ are the model parameters. The values of the calibrated parameters are 
given Table 5. Fig. 13 shows the flow stress at strain rates from $0.005-1600 / \mathrm{s}$ at different strain levels, and the nonlinear increasing trend of shear stress as a function of logarithmic shear strain rate can be quantitatively described by the Cowper-Symonds model.

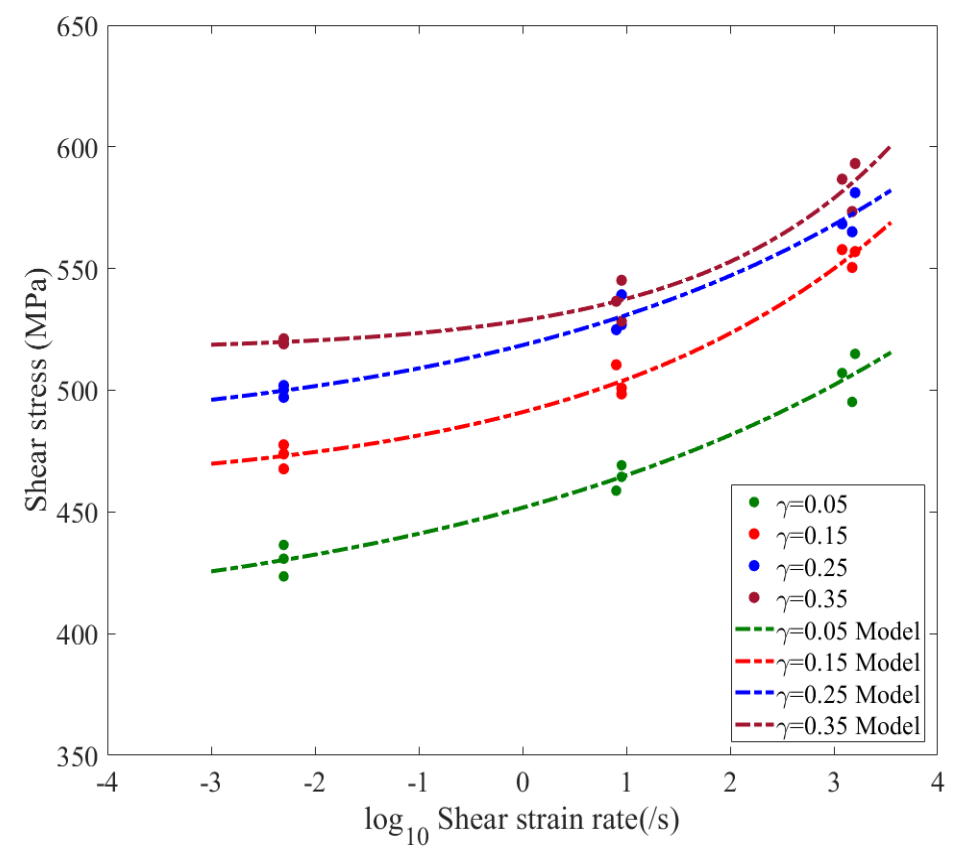

Fig. 13 The shear flow stress as a function of logarithmic shear strain rate at four shear strain levels. The shear strain rate dependent shear stress can be quantitatively described using the Cowper-Symonds model.

\begin{tabular}{|c|c|c|}
\hline Shear Strain & $\mathrm{D}$ & $\mathrm{p}$ \\
\hline 0.05 & $1.262 \mathrm{E} 9$ & 10.554 \\
\hline 0.15 & $5.094 \mathrm{E} 7$ & 6.758 \\
\hline 0.25 & $2.028 \mathrm{E} 9$ & 8.738 \\
\hline 0.35 & $8.231 \mathrm{E} 6$ & 4.276 \\
\hline
\end{tabular}

Table 5 Constants in Cowper-Symonds model

To quantitatively evaluate the strain rate dependent strain hardening, Fig. 14 shows the average strain hardening coefficient (strain hardening modulus) as a function of shear strain from low to high strain rates. The average shear stress-strain relationship 
between shear strain 0.05 and 0.4 is firstly fitted by a two-term power series model using Matlab and the corresponding smooth strain hardening coefficient evolution can be obtained by the differential of the fitted shear stress with respect to shear strain. The decreasing strain hardening coefficient at high strain rates is above $1000 \mathrm{MPa}$ at shear strain 0.05, which is higher than the strain hardening coefficient about 650 MPa-670 MPa under the quasi-static condition. However, the strain hardening coefficient at high strain rate decreases rapidly and becomes smaller than that under quasi-static condition beyond shear strain of 0.15 . The strain hardening coefficient at medium strain rate basically lies between the hardening parameters at the quasi-static condition and high strain rates. Observations of the strain hardening evolutions in Fig.14 indicate the hardening capacity of $\mathrm{Ti} 3 \mathrm{Al} 2.5 \mathrm{~V}$ is apparently strain rate and strain dependent.

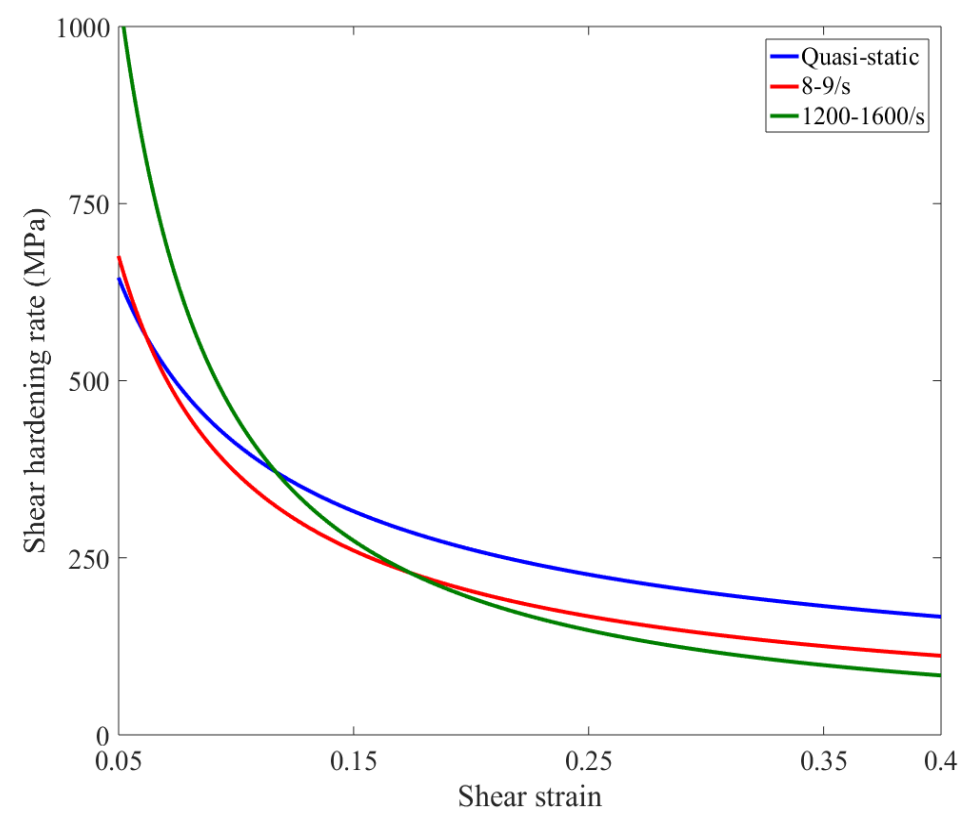

Fig. 14 Average strain hardening coefficient in the engineering shear stress-strain relationship at various strain rates. The strain hardening coefficient is the strain hardening modulus, which is the differential of shear stress with respect to shear strain. Tests at quasi-static, medium and high strain rate three regions are shown in blue, red and green, respectively. 
Fracture surfaces of the torsion specimens were examined under a Carl Zeiss Evo LS15 VP-Scanning Electron Microscope. The fracture surface of the specimen under quasi-static loading condition presents apparent voids and cracks (white circle) in Fig.15a. SEM fractography of the fracture specimen tested at medium strain rate is characterized by similar but larger voids (white circle) along the shear direction as shown in Fig.15b. Different from the fractograph of specimens under quasi-static and medium strain rate loadings, clear elongated dimples (white arrowed) surrounded by severe shear facets are observed in the fracture surface of the specimen loaded at high strain rate of 1600 /s in Fig.15c, which is similar to those reported in Refs. [43, 44].
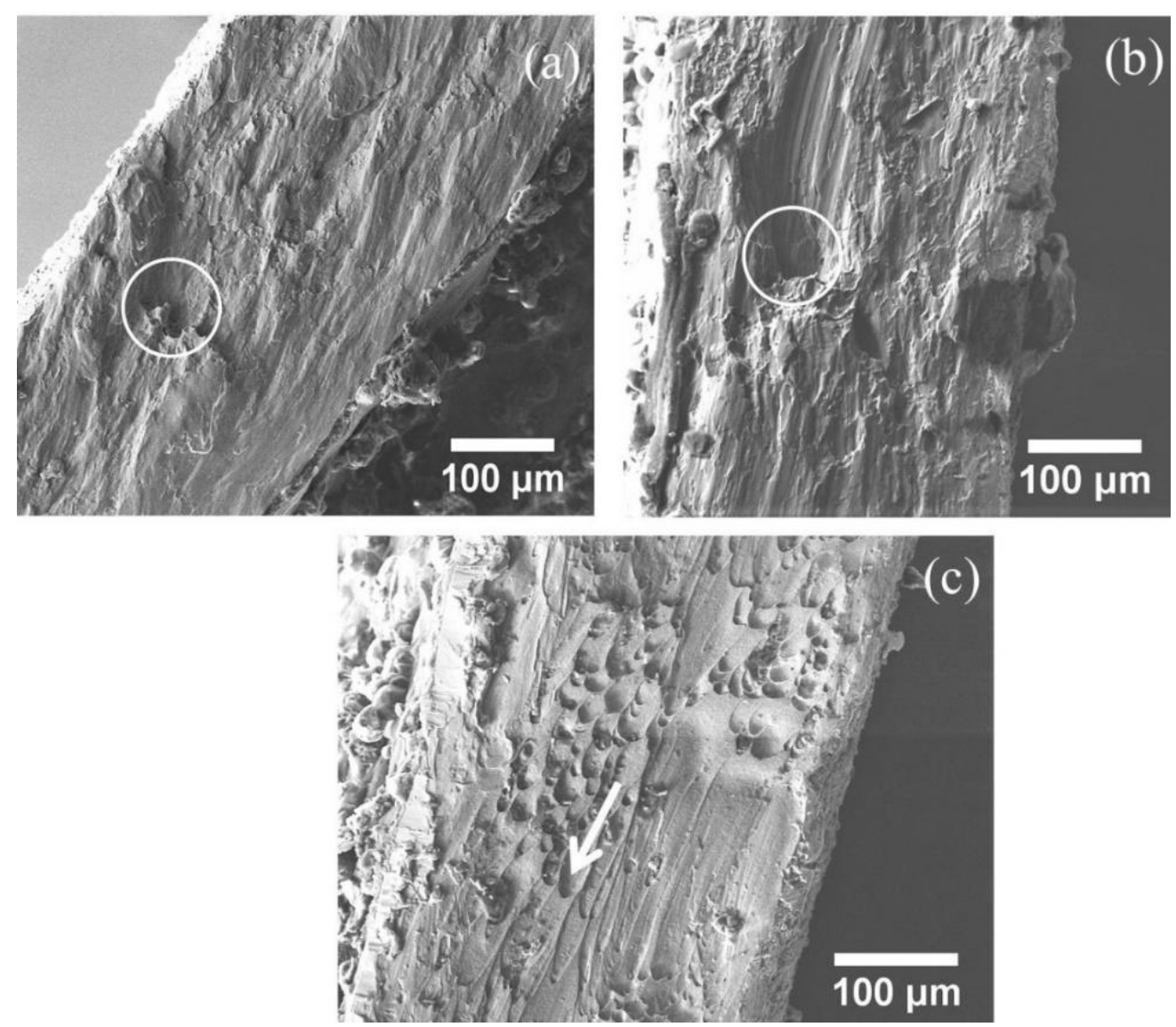

Fig. 15 SEM images of fracture specimens (a) Quasi-static $0.005 / \mathrm{s} \quad$ (b) Medium strain rate $9 / \mathrm{s}$ (c) High strain rate of 1600/s

The dynamic true stress-strain relationship obtained from the torsion tests in the present work along with the compression and SCS tests [34, 35] of the same Ti3Al2.5V alloy is shown in Fig.16. The true stress and true strain from the torsion 
test can be obtained according to Mises stress $\sigma_{\text {true }}=\sqrt{3} \tau$ and the equivalent strain is $\varepsilon_{\text {true }}=\gamma / \sqrt{3}$. As shown in Fig.16, the dynamic yield stress is comparable and approximately $770 \mathrm{MPa}$. At a given true strain 0.20 , the compressive flow stress is $1117 \mathrm{MPa}$ at strain rate of $500 / \mathrm{s}$ and the shear compressive flow stress is $1149 \mathrm{MPa}$ at an equivalent strain rate of $2000 / \mathrm{s}$, while the torsion flow stress is about $1014 \mathrm{MPa}$ at an equivalent strain rate of 866-924/s. Consequently, with the increase of true strain at comparable high strain rates, the compressive and shear compressive flow stress increase significantly, while the torsional stress-strain relationship shows lower strain hardening.

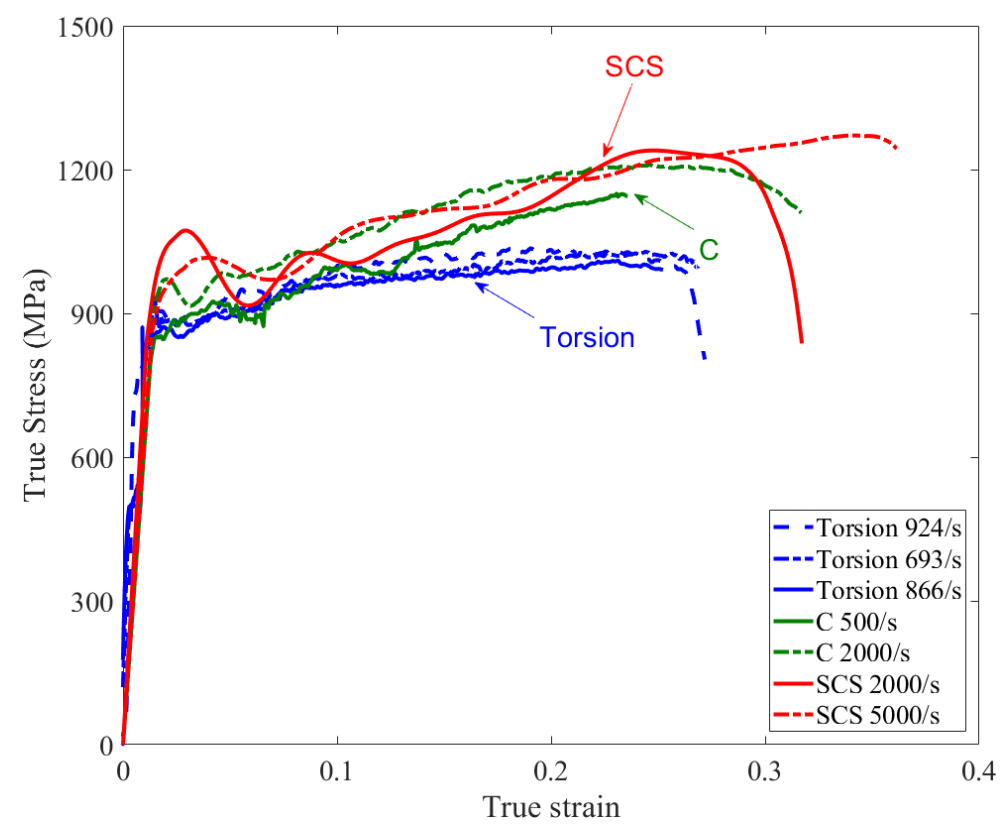

Fig. 16 Comparison of Mises stress-equivalent strain relationship from torsion tests, compression (C) and SCS tests at comparable strain rates reported by Zhang et al. [34, 35]. The present torsional curves are in blue, while the results from compression and SCS are in green and red. 


\section{Discussion}

This paper reports the experimental techniques to characterize the pure shear deformation and failure behaviour from low to high strain rates. The biaxial stress ratio of -1 in torsion test can provide an important connection between shear stress and shear strain relationship, and reveal the fundamental plastic flow response of a material. Most of the previous studies of torsion tests were in the high strain rate region, and limited to Ti6Al4V alloy or pure titanium. This work employs Ti3 Al2.5V as a model material which has been conceived for the design of fan containment in aircraft jet engine application in order to improve the structural impact resistance events, such as blade off or bird strike. This present paper attempts to develop the techniques to characterize pure shear constitutive relationship, by using different loading apparatuses, synchronized with high-speed camera systems, which reveals the local shear stress-strain relationship and provide strain measurement under shear deformation up to fracture.

A single camera would be able to monitor a certain area of the gauge section of torsion specimen, consequently the failure locus may not be observed. A four camera test system was developed to observe the entire specimen gauge section, which enables measurements of the strain field in the failure region. The test machine data has been analysed to obtain a global measurement of shear stress-strain relationship. It is clearly found that this global stress-strain relationship is in line with data obtained by DIC for the flow response of the material. However, this global shear stress-strain data from the test machine, cannot accurately represent the localised failure strain as it represents the global behaviour of specimen. The novel four camera system would enable more accurate measurement of shear stress-strain relationship to be obtained at the failure location with good reproducibility. There are some variations in the strain distribution around the circumference of the specimen test section, which may be due to minor disparities in the test section thickness from the difficulties in machining the specimen and possible misalignment of the test fixture and specimen [23]. 
Meanwhile, the medium strain rate pure shear response, which is rarely reported in the literature, is also studied in order to provide a better understanding of the rate dependency of titanium alloys under pure shear condition. The medium strain rate tests were developed at a strain rate of about 9 /s by using a bespoke designed fixture on the Instron machine. Constant strain rate was achieved in the plastic deformation region. For high rate torsion tests, the Campbell Hopkinson torsion bar was employed which had previously been used for dynamic characterization. Almost constant shear strain rate of $1600 / \mathrm{s}$ can be achieved when the specimen enters the plastic deformation zone. The deformation process at different stages was recorded by a high-speed Kirana camera. The shear stress-strain relationships at medium strain rate and high strain rate show good repeatability. The medium strain rate data is also important to illustrate the non-linear increasing trend of shear stress as a function of shear strain rate, which can be described by Cowper-Symonds material model. Fracture mechanisms of the torsion specimens were examined using SEM. The fracture surfaces of the specimen under quasi-static condition and medium strain rate loading present similar voids and cracks along the shear direction. However, clear elongated dimples surrounded by severe shear facets are observed in the fracture surface of the specimen loaded at high strain rates, similar to those dynamic shear fracture mechanisms observed in Refs. [43, 44], in which adiabatic shear banding is the typical dynamic failure mode associated with high temperature rise due to the thermomechanical coupling effect [45].

A comparison of the response of the torsion specimen and the SCS specimen [24-26] under complex stress state conditions would be of interest. Although there is a scarcity of results on the dynamic response of the present investigated alloy, the equivalent stress-strain relationship obtained from the present torsion tests, compression and SCS tests of the same Ti3A12.5V alloy recently reported by Zhang et al. [34, 35] can be compared at comparable strain rates. The dynamic yield stress values are similar, however, the flow stress from compression and SCS increase with the increasing equivalent strain, compared to that in dynamic torsional stress-strain relationship with lower strain hardening. 
Due to the limitation of space and equipment, it's impossible to employ a four camera system for the medium and high strain rate tests on the current Instron machine and Hopkinson torsion bar. Further work will consider using a three or four camera system for medium and high strain rate tests, in order to better characterize the deformation and failure process of the specimen, in the spirit of Duffy [31, 46]. Given the high temperature working environment of aircraft jet engine, torsion tests at high temperature will be studied as the next step and the corresponding techniques will be developed. Likewise, the present torsion technique will contribute to the further development of Split Hopkinson tension-torsion bar apparatus for the understanding of rate-dependent behavior of a material under complex stress states [47].

\section{Conclusion}

This paper reports the experimental techniques to obtain the torsional constitutive response, using a screw driven Zwick test machine, a hydraulic Instron machine, and a Campbell torsion Hopkinson bar synchronized with a high-speed camera over a wide range of strain rates. A near alpha Ti3Al2.5V alloy is chosen as a model material in this study. The conclusions are as follows:

- A series of consistent results of pure shear stress-strain relationships have been obtained from the proposed measurement techniques.

- A novel four camera digital image analysis system has been employed to observe the material deformation and failure during low rate torsion tests. This enabled shear stress-strain response to be obtained at the location of the onset of material failure with good reproducibility.

- The medium strain rate data, which cannot be easily achieved with conventional apparatus and specimen, is essential to reveal shear stress as a function of logarithm shear strain rate. Strain rate dependent behaviour was observed for the titanium alloy under shear loading, typically with a nonlinear increase in flow stress with loading rate. The Cowper-Symonds material model is able to describe the corresponding rate dependency. 
- In general, the strains at which failure commences in the test specimens decreases with increasing loading rate.

- Observations of the strain hardening rate evolution indicate the hardening capacity of Ti3A12.5V is also strain and strain rate dependent.

- Dynamic torsional stress-strain relationship shows low strain hardening, compared to the response from compression or SCS test which shows a significant increase in the flow stress with the increasing true strain.

\section{Acknowledgements}

The authors thank Dr. M. Rutherford in ARM Ltd., Mr. J. Fullerton, Mr. S. Carter, Mrs. K. Bamford, Dr. K. Dragnevski and Dr. M. Tkaczyk for their assistance. The fruitful discussions with Dr. DAS. Macdoguall, Dr. J. Reed from Rolls-Royce plc, Prof. D. Eakins during the development of current torsion technique, about the use of tubular specimen and the understanding of pure shear failure mechanism are appreciated. Prof. D. Rittel and Dr. A. Dorogoy at Technion-Israel Institute of Technology are acknowledged for the long term guidance and discussion on the SCS techniques.

\section{Appendix A:}

Shear strain from the cylindrical gauge section is projected onto a flat image plane by the DIC imaging system. During shear deformation, a point a on the cylinder surface travels with the rotation angle $\theta$ through an arc $\mathrm{S}$ to a new location $\mathrm{b}$. Likewise, this movement on the image plane is horizontal over a length $L$ from the point $c$ to $d$, as illustrated in Fig. A1 with the mentioned points.

The shear strain from the cylinder analysis is given by

$$
\gamma_{\text {cylinder }}=\frac{\mathrm{R} \theta}{l}
$$

The shear strain from the image plane from DIC analysis is given by

$$
\gamma_{\text {plane }}=\frac{L}{l}
$$

where $\mathrm{L}=\mathrm{R} \sin \theta, l$ is the length of gauge section. 


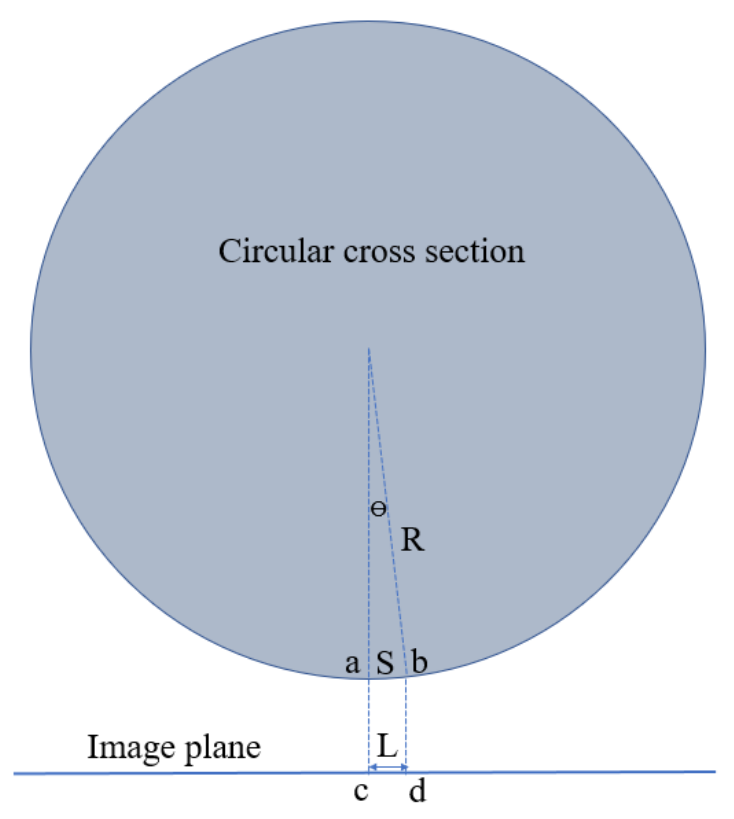

Fig. A1 Schematic of a circular cross section through a torsion specimen gauge.

Fig. A2 compares the calculated shear strain on the image plane and cylinder surfaces. The error between two shear strain values increases to $0.7 \%$ at shear strain 0.75 from the cylinder surface. The largest shear strains observed the current tests were less than 0.75. Therefore, no correction to the measured DIC strains were made in the present work. 


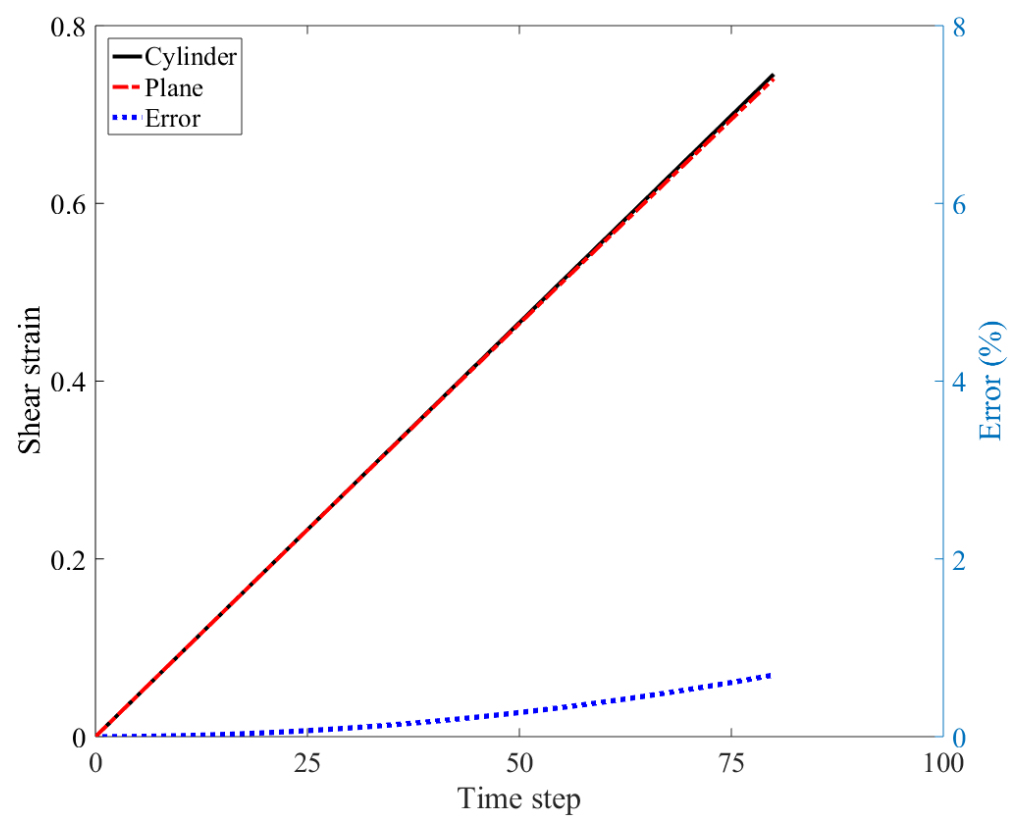

Fig. A2 Difference of shear strain from DIC image plane and cylinder surface.

\section{References:}

[1] B. Hopkinson, A method of measuring the pressure produced in the detonation of high explosives or by the impact of bullets, Proceedings of the Royal Society of London. Series A, 89 (1914) 411-413.

[2] H. Kolsky, An investigation of the mechanical properties of materials at very high rates of loading, Proceedings of the Physical Society. Section B, 62 (1949) 676.

[3] J. Harding, E. Wood, J. Campbell, Tensile testing of materials at impact rates of strain, J. Mech. Eng. Sci, 2 (1960) 88-96.

[4] S. Nemat-Nasser, J.B. Isaacs, J.E. Starrett, Hopkinson techniques for dynamic recovery experiments, Proceedings of the Royal Society of London. Series A: Mathematical and Physical Sciences, 435 (1991) 371-391.

[5] J. Field, S. Walley, W. Proud, H. Goldrein, C. Siviour, Review of experimental techniques for high rate deformation and shock studies, Int. J. Impact Eng, 30 (2004) 725-775.

[6] W.W. Chen, B. Song, Split Hopkinson (Kolsky) bar: design, testing and applications, Springer Science \& Business Media, 2010.

[7] H. Espinosa, A. Patanella, M. Fischer, A novel dynamic friction experiment using a modified Kolsky bar apparatus, Exp Mech, 40 (2000) 138-153. 
[8] P. Li, C.R. Siviour, N. Petrinic, The Effect of Strain Rate, Specimen Geometry and Lubrication on Responses of Aluminium AA2024 in Uniaxial Compression Experiments, Exp Mech, 49 (2009) 587-593.

[9] F. Lu, Y. Lin, X. Wang, L. Lu, R. Chen, A theoretical analysis about the influence of interfacial friction in SHPB tests, Int. J. Impact Eng, 79 (2015) 95-101.

[10] G. Yang, Y. Song, The TSHB Technique for Material Testing at High Rates of Strain, Applied Mathematics and Mechanics, 5 (1985).

[11] W.E. Baker, C.H. Yew, Strain-Rate Effects in the Propagation of Torsional Plastic Waves, Journal of Applied Mechanics, 33 (1966) 917-923.

[12] J. Campbell, A. Dowling, The behaviour of materials subjected to dynamic incremental shear loading, J Mech Phys Solids, 18 (1970) 43-63.

[13] J. Lewis, J. Campbell, The development and use of a torsional Hopkinson-bar apparatus, Exp Mech, 12 (1972) 520-524.

[14] J. Duffy, J.D. Campbell, R.H. Hawley, On the Use of a Torsional Split Hopkinson Bar to Study Rate Effects in 1100-0 Aluminum, Journal of Applied Mechanics, 38 (1971) 83-91.

[15] T. Nicholas, J.E. Lawson, On the determination of the mechanical properties of materials at high shear-strain rates, J Mech Phys Solids, 20 (1972) 57-62.

[16] T. Nicholas, J. Campbell, Shear-strain-rate effects in a high-strength aluminum alloy, Exp Mech, 12 (1972) 441-447.

[17] J.E. Lawson, T. Nicholas, The dynamic mechanical behavior of titanium in shear, J Mech Phys Solids, 20 (1972) 65-76.

[18] R. Fadida, D. Rittel, A. Shirizly, Dynamic Mechanical Behavior of Additively Manufactured Ti6Al4V With Controlled Voids, Journal of Applied Mechanics, 82 (2015).

[19] M. Garg, A.D. Mulliken, M.C. Boyce, Temperature Rise in Polymeric Materials During High Rate Deformation, Journal of Applied Mechanics, 75 (2008).

[20] W. Chen, F. Lu, D.J. Frew, M.J. Forrestal, Dynamic Compression Testing of Soft Materials, Journal of Applied Mechanics, 69 (2002) 214-223. 
[21] P. Li, N. Petrinic, C.R. Siviour, R. Froud, J.M. Reed, Strain rate dependent compressive properties of glass microballoon epoxy syntactic foams, Materials Science and Engineering: A, 515 (2009) 19-25.

[22] J.P. Hou, C. Ruiz, A. Trojanowski, Torsion tests of thermosetting resins at impact strain rate and under quasi-static loading, Materials Science and Engineering: A, 283 (2000) 181-188.

[23] N.A. Fellows, J. Harding, Use of high-speed photography to study localisation during high-strain-rate torsion testing of soft iron, Materials Science and Engineering: A, 298 (2001) 90-99.

[24] D. Rittel, S. Lee, G. Ravichandran, A shear-compression specimen for large strain testing, Exp Mech, 42 (2002) 58-64.

[25] D. Rittel, G. Ravichandran, S. Lee, Large strain constitutive behavior of OFHC copper over a wide range of strain rates using the shear compression specimen, Mechanics of Materials, 34 (2002) 627-642.

[26] A. Dorogoy, D. Rittel, A. Godinger, Modification of the Shear-Compression Specimen for Large Strain Testing, Exp Mech, (2015) 1-13.

[27] D. Rittel, L. Zhang, S. Osovski, Mechanical Characterization of Impact-Induced Dynamically Recrystallized Nanophase, Physical Review Applied, 7 (2017) 044012.

[28] C. Leyens, M. Peters, Titanium and titanium alloys: fundamentals and applications, John Wiley \& Sons, 2003.

[29] D. Macdougall, J. Harding, The measurement of specimen surface temperature in high-speed tension and torsion tests, Int. J. Impact Eng, 21 (1998) 473-488.

[30] D. Macdougall, J. Harding, A constitutive relation and failure criterion for Ti6Al4V alloy at impact rates of strain, J Mech Phys Solids, 47 (1999) 1157-1185.

[31] S. Liao, J. Duffy, Adiabatic shear bands in a Ti-6Al-4V titanium alloy, J Mech Phys Solids, 46 (1998) 2201-2231.

[32] D.R. Chichili, K. Ramesh, K.J. Hemker, Adiabatic shear localization in $\alpha$-titanium: experiments, modeling and microstructural evolution, J Mech Phys Solids, 52 (2004) 1889-1909. 
[33] R. Yang, H. Zhang, L. Shen, Y. Xu, Y. Bai, B. Dodd, A modified split Hopkinson torsional bar system for correlated study of $\tau-\gamma$ relations, shear localization and microstructural evolution, Philosophical Transactions of the Royal Society of London A: Mathematical, Physical and Engineering Sciences, 372 (2014) 20130208.

[34] L. Zhang, D. Rittel, S. Osovski, Thermo-mechanical characterization and dynamic failure of near $\alpha$ and near $\beta$ titanium alloys, Mat Sci Eng a-Struct, 729 (2018) 94-101.

[35] L. Zhang, A. Pellegrino, D. Townsend, N. Petrinic, Thermomechanical Constitutive Behaviour of a Near $\alpha$ Titanium Alloy over a Wide Range of Strain Rates: Experiments and Modelling, International Journal of Mechanical Sciences, 189 (2020) 105970.

[36] S. Tzibula, Z. Lovinger, D. Rittel, Dynamic tension of ductile polymers: Experimentation and modelling, Mechanics of Materials, 123 (2018) 30-42.

[37] L. Zhang, A. Pellegrino, D. Townsend, N. Petrinic, Strain rate and temperature dependent strain localization of a near $\alpha$ titanium alloy, Int. J. Impact Eng, 145 (2020) 103676.

[38] A. Pellegrino, V.L. Tagarielli, R. Gerlach, N. Petrinic, The mechanical response of a syntactic polyurethane foam at low and high rates of strain, Int. J. Impact Eng, 75 (2015) 214-221.

[39] T. Bhujangrao, C. Froustey, E. Iriondo, F. Veiga, P. Darnis, F.G. Mata, Review of Intermediate Strain Rate Testing Devices, Metals, 10 (2020) 894.

[40] L. Zhang, A. Pellegrino, D. Townsend, N. Petrinic, Temperature Dependent Dynamic Strain Localization and Failure of Ductile Polymeric Rods under Large Deformation, International Journal of Mechanical Sciences, 204 (2021) 106563.

[41] D. Rittel, On the conversion of plastic work to heat during high strain rate deformation of glassy polymers, Mechanics of Materials, 31 (1999) 131-139.

[42] G.R. Cowper, P.S. Symonds, Strain-hardening and strain-rate effects in the impact loading of cantilever beams, in: Brown Univ. Tech. Rept. No. 28, 1957. 
[43] D. Rittel, Z. Wang, Thermo-mechanical aspects of adiabatic shear failure of AM50 and Ti6Al4V alloys, Mechanics of materials, 40 (2008) 629-635.

[44] M. Zhou, A.J. Rosakis, G. Ravichandran, Dynamically propagating shear bands in impact-loaded prenotched plates-I. Experimental investigations of temperature signatures and propagation speed, J Mech Phys Solids, 44 (1996) 981-1006.

[45] D. Rittel, L.H. Zhang, S. Osovski, The dependence of the Taylor-Quinney coefficient on the dynamic loading mode, J Mech Phys Solids, 107 (2017) 96-114.

[46] A. Marchand, J. Duffy, An experimental study of the formation process of adiabatic shear bands in a structural steel, J Mech Phys Solids, 36 (1988) 251-283.

[47] Y. Xu, L. Farbaniec, C. Siviour, D. Eakins, A. Pellegrino, The Development of Split Hopkinson Tension-Torsion Bar for the Understanding of Complex Stress States at High Rate, in: Dynamic Behavior of Materials, Volume 1: Proceedings of the 2020 Annual Conference on Experimental and Applied Mechanics, Springer International Publishing, 2021, pp. 89-93. 